\title{
Inmunoglobulinas en sepsis y shock séptico
}

\author{
JESSICA SALINAS L. y ALBERTO FICA C.
}

\section{Immunoglobulins in sepsis and septic shock}

Clinical efficacy of polyspecific immunoglobulins or monoclonal antibodies to treat patients with severe sepsis or septic shock is still under debate after several clinical trials. Only a few of them have been able to demonstrate a direct benefit to reduce mortality or this effect appears after meta-analysis. Evidence sustains that polyspecific immunoglobulin $\mathrm{G}$ reduces mortality in these patients, being this effect higher for IgM-enriched immunoglobulins. Best indications are postsurgical sepsis or early septic shock patients with high titers of endotoxinemia. The use of intravenous immunoglobulins is also recommended for the treatment of patients with streptococcal toxic shock, as demonstrated by the evidence obtained through case-control studies and one randomized clinical trial with a clear trend toward benefit.Evidence does not sustain a favorable impact on mortality for monoclonal antibodies directed against bacterial lypopolysaccaride, other bacterial antigens or against TNF- $\alpha$. Furthermore, infusion of recombinant IL-1 receptor antagonist or soluble receptors for TNF- $\alpha$ that could attenuate the inflammatory response have not demonstrated utility after many clinical trials. These therapeutic tools are characterized by a high acquisition cost and adequate cost-effectiveness analysis has not been yet performed.

Key words: intravenous immunoglobulin; sepsis; septic shock; lypopolysaccaride; monoclonal antibodies; antiTNF- $\alpha$ antibodies; TNF- $\alpha$ soluble receptor fusion proteins; Interleukin 1 receptor antagonist.

Palabras clave: inmunoglobulina intravenosa; sepsis; shock séptico; lipolisacáridos; anticuerpos monoclonales; anticuerpos 1anti FNT- $\alpha$; proteínas de fusión del receptor soluble de FNT- $\alpha$; antagonista del receptor de interleukina 1

\section{Introducción}

El síndrome séptico es una causa importante de morbilidad y mortalidad, con una letalidad promedio de $40 \%$, que se incrementa más allá del $60 \%$ en pacientes con shock séptico. En este síndrome, la respuesta inflamatoria sistémica es iniciada por la liberación de componentes microbianos, incluyendo endotoxinas, peptidoglicanos, ácido teicoico y varias exotoxinas. Estos componentes estimulan una respuesta inflamatoria concertada y en cascada, mediada por citoquinas que incluyen al factor de necrosis tumoral alfa (FNT- $\alpha$ ), interleuquina-1 (IL-1) e interleuquina6 (IL-6). Los dos primeros inducen hipotensión arterial, siendo la combinación de ellos más potente que su acción aislada. Las tres citoquinas mencionadas se encuentran en concentraciones aumentadas en el suero de pacientes con shock séptico $^{1}$. En esta respuesta participan también, proteínas sintetizadas en forma natural por los macrófagos, tales como el antagonista del receptor de IL-1 (IL-1ra), de efecto anti-inflamatorio.

El advenimiento de inmunoglobulinas purificadas desde un pool de numerosos donantes (inmunoglobulinas poliespecíficas) y la capacidad de producir anticuerpos monoclonales específicos, han permitido contar con nuevas herramientas terapéuticas en pacientes con sepsis y shock séptico, las que han tenido como propósito bloquear la cascada inflamatoria en sus inicios o en su desarrollo. En las últimas décadas, se han desarrollado numerosos estudios con estos preparados en pacientes con sepsis, obteniendo sin

Departamento de Medicina, Hospital Clínico Universidad de Chile. Santiago, Chile.

Sección de Inmunología (JSL).

Sección de Infectología (AFC).

Recibido: 17 noviembre 2003

Aceptado: 27 octubre 2004 
embargo, resultados discordantes que hacen necesario revisar las virtudes reales de estas intervenciones. En este documento se analizan las alternativas farmacéuticas actualmente disponibles, sus mecanismos de acción, reacciones adversas y administración de estos preparados, y finalmente las evidencias de su eficacia cuando son administrados con fines terapéuticos.

Alternativas disponibles. Cuatro modalidades de intervención se han explorado en estos pacientes: inmunoglobulinas purificadas, anticuerpos monoclonales contra estructuras bacterianas, anticuerpos monoclonales contra mediadores de la respuesta inmune y antagonistas de receptores de citoquinas (Tabla 1).

De acuerdo al tipo de preparado, estas intervenciones permiten la neutralización de productos bacterianos, la modulación de la respuesta inmune o la interrupción o una limitación de la cascada inflamatoria (Tabla 1).

\section{Características y mecanismo de acción}

\section{Inmunoglobulinas poliespecíficas}

Las inmunoglobulinas poliespecíficas son productos de uso intravenoso, de naturaleza heterogénea, preparados a partir de un pool de 10.000 a 20.000 donantes humanos. Contienen más de 90\% de inmunoglobulina $\mathrm{G}$ ( $\mathrm{IgG}$ ) en forma monomérica, poliespecíficas, con una distribución normal de subclases, libre de agregados, cantidades mínimas de las otras inmunoglobulinas y están libres de agentes infecciosos ${ }^{2}$.

Los preparados de inmunoglobulinas poliespecíficas difieren en el tipo de inmunoglobulinas predominante y se clasifican básicamente en dos grandes grupos. Aquellos que contienen $\mathrm{IgG}$ (IGIV) y aquellos enriquecidos con IgM y que también contienen IgA (IGMAIV).
El riesgo de transmisión de $\mathrm{VIH}$, virus $\mathrm{B}$ o C de hepatitis u otros agentes de transmisión sanguínea es prácticamente nulo con los preparados actuales. Esto debido a la selección estricta de donantes seronegativos, y a los procedimientos físicos y químicos de inactivación viral posteriores: fraccionamiento con etanol y frío, filtración, tratamiento con solventes y/o detergentes (ácido caprílico y $\beta$-propionolactona), inactivación fotoquímica, precipitación en polietilenglicol, cromatografía de intercambio iónico y pasteurización.

Mecanismo de acción. En el caso de la sepsis y el shock séptico, su utilidad está dada por dos mecanismos esenciales: acción antimicrobiana y modulación de la respuesta inflamatoria ${ }^{3}$ (Tabla 2).

Los preparados comerciales de IGIV ejercen una acción antimicrobiana directa a través de anticuerpos neutralizantes y opsonizantes contra bacterias, endotoxinas o exotoxinas de microorganismos específicos, que permiten aumentar su depuración hepática o esplénica. Estas incluyen anticuerpos contra Campylobacter jejuni, Yersinia enterocolitica, Staphylococcus aureus (incluso resistente a meticilina), Enterococcus faecalis (también resistente a vancomicina), Streptococcus $\beta$ hemolíticos, Pseudomonas aeruginosa y anticuerpos contra el lipopolisacárido (LPS), la toxina alfa de $S$. aureus y algunos superantígenos de Streptococcus pyogenes. La acción antimicrobiana se ejerce también mediante la estimulación de la producción de especies reactivas del oxígeno, en leucocitos y de la actividad bactericida del suero (Tabla 2).

Los mecanismos de modulación de la respuesta inflamatoria propuestos, contemplan básicamente la interferencia con el efecto de citoquinas, la modulación de la actividad de linfocitos B y T (CD4+ y CD8+), y la prevención de una excesiva activación del complemento ${ }^{4}$.

Tabla 1. Modalidades de intervención en sepsis y shock séptico con inmunoglobulinas y productos relacionados

\begin{tabular}{|c|c|}
\hline Modalidad & Ejemplos y comentarios \\
\hline $\begin{array}{l}\text { - Inmunoglobulina } \mathrm{G} \text { poliespecífica enriquecida } \\
\text { o no con IgM }\end{array}$ & $\begin{array}{l}\text { Intervención de amplio espectro que permite antagonizar bac- } \\
\text { terias o sus componentes y modular la respuesta inmune }\end{array}$ \\
\hline $\begin{array}{l}\text { - Anticuerpos monoclonales contra estructuras } \\
\text { bacterianas }\end{array}$ & $\begin{array}{l}\text { Dirigidos contra componentes comunes de bacilos gramnega- } \\
\text { tivos, sin cobertura sobre otros tipos de microorganismos }\end{array}$ \\
\hline $\begin{array}{l}\text { - Anticuerpos monoclonales contra mediadores } \\
\text { de la respuesta inmune }\end{array}$ & $\begin{array}{l}\text { Dirigidos a interrumpir la cascada inflamatoria mediante } \\
\text { anticuerpos contra FNT- } \alpha\end{array}$ \\
\hline $\begin{array}{l}\text { - Antagonistas de receptores de citoquinas } \\
\text { específicas }\end{array}$ & $\begin{array}{l}\text { Dirigidos a interrumpir la respuesta inflamatoria utilizando } \\
\text { moléculas recombinantes similares a las sintetizadas en forma } \\
\text { natural }\end{array}$ \\
\hline
\end{tabular}


Tabla 2. Mecanismos potenciales de acción de inmunoglobulinas poliespecíficas en pacientes con sepsis

Inactivación de toxinas

- Neutralización de endotoxinas bacterianas, exotoxinas y proteínas de membrana externa de BGN*

- Aumento de la depuración de LPS**

- Aumento de la captación hepática y esplénica de LPS

- Atenuación de la adherencia celular bacteriana, invasión celular e invasión de órganos

\section{Estimulación de leucocitos y actividad bactericida del suero}

- Incremento en la producción de radicales libres de oxígeno en neutrófilos y de la fagocitosis por neutrófilos ante E. coli lograda por IGIV en pacientes con sepsis por BGN

- Aumento de la respuesta oxidativa inducida por LPS en neutrófilos por IGIV*** y atenuación por IGMAIV****

- Aumento de la actividad opsónica del suero

- Neutralización de la mitogenicidad bacteriana

- Aumento de la función fagocítica de las células de Kupffer durante la fase tardía de la sepsis y endotoxemia

- Freno al aumento de la actividad fagocítica de los neutrófilos en RN de pretérmino ante una infección por Streptococcus agalactiae

\section{Interferencia con el efecto de las citoquinas}

- Modulación de la liberación de citoquinas y antagonistas de las citoquinas desde células mononucleares sanguíneas estimuladas por endotoxinas o superantígenos, disminuyendo la actividad pro-inflamatoria y aumentando la actividad anti-inflamatoria.

- Presencia de citoquinas inmunomoduladoras en el preparado de IVIG (TGF- $\beta$ )

- Neutralización de citoquinas por auto-anticuerpos anti-citoquina en preparaciones de IVIGs

\section{Prevención de una excesiva activación del complemento}

- Altos niveles de IgG impiden la unión de C3 y C4 a las células blanco, modulando la injuria tisular

*: bacilos gramnegativos; ** lipopolisacárido; IGIV: inmunoglobulina intravenosa tipo IgG; IGMAIV: inmunoglobulina intravenosa que contiene IgG e IgM;TGF- $\beta$ : factor de crecimiento transformante beta.

(Adaptado de ref. 3)

La modulación permite una disminución de citoquinas pro-inflamatorias como IL-6, IL-1 y FNT- $\alpha$, y el aumento del receptor antagonista de la IL-1 (IL-1ra), favoreciendo un estado antiinflamatorio. Se ha evidenciado además la presencia en los preparados de IGIV, de anticuerpos anti-citoquinas como la IL-1, IL-6, FNT- $\alpha$ e interferones ${ }^{4,5}$.

Los preparados enriquecidos con IgM presentan una mayor inhibición de la activación excesiva del complemento comparados a las IGIV y han estado asociado en ciertos estudios a una mayor eficacia clínica (ver más adelante).

\section{Anticuerpos monoclonales}

Estas inmunoglobulinas han sido en general preparadas con una especificidad dirigida hacia endotoxinas, otros compuestos bacterianos o hacia citoquinas involucradas en la respuesta inflamatoria. En el primer caso están los anticuerpos E5 y HA-1A dirigidos contra el lípido A del LPS, una estructura común a los bacilos gramnegativos claramente involucrada en el fenómeno de respuesta inflamatoria sistémica. También se han diseñado anticuerpos dirigidos contra antígenos comunes a enterobacterias ${ }^{6}$. En el caso de las citoquinas, estos han estado dirigidos contra el factor de necrosis tumoral (anti-FNT- $\alpha$ : infliximab $)^{7}$ (Tabla 3).

\section{Antagonistas de citoquinas}

Las intervenciones en sepsis involucran también a moléculas recombinantes antagonistas del efecto de citoquinas específicas. Una de ellas es el antagonista natural del receptor de la IL-1, sintetizado durante la infección natural (IL-1ra: IL-1 "receptor antagonista") y que permite limitar la respuesta inflamatoria ${ }^{1,8}$. Otras moléculas incluyen los receptores solubles del FNT- $\alpha$ (por ejemplo etarnecept) que también permiten neutralizar el efecto de esta citoquina y limitar la respuesta inmune.

Los receptores solubles del FNT son liberados desde la membrana celular por proteolisis durante una infección y permiten neutralizar al FNT- $\alpha$. $\mathrm{Al}$ menos dos de estos receptores con diferentes pesos moleculares han sido descritos y ambos han sido sintetizados como moléculas recombinantes unidas a una fracción Fc de IgG huma$\mathrm{na}^{9-12}$ (Tabla 4). 
Tabla 3. Anticuerpos monoclonales en sepsis

\begin{tabular}{ll}
\hline Tipo de anticuerpo & Comentario \\
\hline • E5 & $\begin{array}{l}\text { Anticuerpo monoclonal IgM murino preparado con una variante } \\
\text { rugosa de E. coli, desprovista de la cadena de polisacáridos ligada } \\
\text { al lípido A (que facilita la estimulación antigénica). Esto permite } \\
\text { dirigir anticuerpos contra un componente patogénico común en } \\
\text { los bacilos gramnegativos }\end{array}$ \\
• HA-1A & $\begin{array}{l}\text { Anticuerpo monoclonal similar al anterior pero con la porción Fc } \\
\text { humana que permite una menor sensibilización ("humanizado") }\end{array}$ \\
- Anti-antígeno común de enterobacterias & $\begin{array}{l}\text { Anticuerpo monoclonal murino dirigido a un glicofosfolípido de } \\
\text { superficie común a la familia Enterobacteriaceae }\end{array}$ \\
& $\begin{array}{l}\text { Anticuerpo monoclonal murino contra FNT-a. También se ha uti- } \\
\text { lizado sólo el fragmento F(ab') del anticuerpo murino para redu- } \\
\text { cir su inmunogenicidad, facilitar su penetración en tejidos y mini- } \\
\text { mizar la interacción con los receptores Fc }\end{array}$ \\
\hline
\end{tabular}

Tabla 4. Antagonistas de citoquinas en sepsis

\begin{tabular}{ll}
\hline Antagonista & Comentario \\
\hline - IL-1 ra: antagonista del receptor de IL-1 & $\begin{array}{l}\text { Molécula recombinante destinada a antagonizar el efecto de IL-1 } \\
\text { en sus receptores naturales. Efecto anti-inflamatorio }\end{array}$ \\
- Proteínas de fusión del receptor & $\begin{array}{l}\text { Proteína de fusión de la porción Fc de IgG humana con alguno de los } \\
\text { dos receptores solubles de FNT descritos. Efecto anti-inflamatorio } \\
\text { por antagonismo competitivo concentración-dependiente con los } \\
\text { receptores de membrana }\end{array}$ \\
\hline
\end{tabular}

\section{Riesgos y efectos adversos de las inmunoglobulinas poliespecíficas, anticuerpos monoclonales y antagonistas de citoquinas}

Las reacciones adversas a estos preparados son infrecuentes y rara vez severas, lo que permite que estos productos sean considerados como medicamentos seguros.

Reacciones adversas asociadas a inmunoglobulinas poliespecíficas. Se ha estimado que las reacciones adversas provocadas por estos preparados son de muy baja magnitud y que las reacciones severas o letales son improbables.

Las complicaciones de la terapia con IGIV pueden clasificarse en no-anafilácticas y anafilácticas. Las primeras incluyen calofríos, cefalea, dorsalgia, malestar general, fiebre, rash cutáneo, náuseas, parestesias, hipo o hipertensión y sobrecarga de volumen. La mayoría se relaciona a la velocidad de infusión, se presentan dentro de los primeros 30 minutos de la administración y son manejadas suspendiendo temporalmente la infusión, reduciendo su velocidad o con pre- medicación. Complicaciones más raras son la meningitis aséptica y la falla renal aguda. Las reacciones anafilácticas pueden verse en los pacientes que presentan un déficit absoluto de IgA, en los cuales la administración de IGIV con altos niveles de IgA puede inducir la síntesis de IgE específicas anti-IgA. En estos casos, las reacciones no se presentan con la primera dosis, aunque pueden llegar hasta el shock anafiláctico con administraciones posteriores. Para el tratamiento de estos pacientes, deben usarse preparados comerciales con la menor cantidad posible de $\operatorname{Ig} \mathrm{A}^{2}$.

Efectos adversos de los anticuerpos monoclonales. En los ensayos disponibles, los efectos adversos observados con estos compuestos han sido infrecuentes y en general no superiores a los observados con placebo. La frecuencia de estos eventos no supera el 2 a $3 \%$ e incluyen rash alérgico, enfermedad del suero, hipotensión arterial y ocasionalmente shock anafiláctico ${ }^{13-15}$. Las reacciones no anafilácticas revierten fácilmente al suspender la terapia aunque pueden requerir antiinflamatorios no esteroidales o corticoesteroides. 
La mayor parte de los pacientes (90\%) que han recibido anticuerpos monoclonales de origen murino desarrollan anticuerpos contra el preparado durante el seguimiento ${ }^{16}$. Se desconoce si ello puede generar una mayor frecuencia de efectos adversos durante nuevas exposiciones.

Debido a la participación del FNT- $\alpha$ en la respuesta inmune contra patógenos intracelulares tales como Listeria sp, micobacterias y ciertos hongos, el bloqueo del FNT- $\alpha$ puede favorecer la aparición o reactivación de infecciones por patógenos intracelulares. En pacientes tratados con moléculas destinadas a este fin, ya sea con infliximab o etarnecept, no se ha observado una mayor frecuencia de estos eventos en pacientes con sepsis en comparación a lo observado en el grupo placebo ${ }^{10,13}$. No obstante, este riesgo está descrito en pacientes con enfermedad de Crohn o artritis reumatoidea sometidos a terapias con estos anticuerpos, donde se ha observado un incremento de casos de tuberculosis luego de iniciar la terapia ${ }^{17,18}$. La prolongada vida media de infliximab (10 días) y de su efecto biológico (2 meses) explica este riesgo.

Efectos adversos de los antagonistas de citoquinas. Las moléculas recombinantes (por ejemplo IL-1ra), son similares a las moléculas naturales y por ello no han estado asociadas a efectos adversos importantes ${ }^{1,8}$. La molécula de fusión p55-IgG tampoco se ha asociado a una mayor frecuencia de efectos adversos en comparación a placebo ${ }^{9}$. No se han reportado casos de anafilaxia, urticaria o broncoespasmo para estos preparados aunque sí eventos de rash? Como se ha mencionado, no se ha reportado una mayor frecuencia de infecciones por patógenos intracelulares en los pacientes tratados con la molécula de fusión p55-IgG ${ }^{10}$.

\section{Esquemas de dosificación}

Se ha demostrado que los niveles de inmunoglobulinas $\operatorname{IgG}$ e IgM en pacientes con sepsis están severamente deprimidos. Las dosis utilizadas en diferentes ensayos han sido diseñadas para permitir un retorno de sus concentraciones a valores fisiológicos durante varios días. En la Tabla 5 se presentan las dosis habituales utilizadas con fines terapéuticos para las condiciones más aceptadas actualmente (ver más adelante) ${ }^{3}$.

Las dosis de los anticuerpos monoclonales y de moléculas recombinantes han sido derivadas de los resultados de estudios en animales y de las titulaciones desarrolladas en ensayos en seres humanos.
Sólo dos productos de inmunoglobulinas se encuentran actualmente disponibles en Chile (Pentaglobin ${ }^{\circledR}$ y Flebogamma $\left.{ }^{\circledR}\right)$. Otros productos han sido discontinuados (Sandoglobulina ${ }^{\circledR}$, Octagam(®). El costo de adquisición es inferior para el preparado enriquecido en IgM (Tabla 5).

Diferencias de actividad entre preparados. $\mathrm{La}$ estandarización actual de las técnicas de fraccionamiento y obtención de preparados de IGIV, permiten que todas posean una actividad similar, con una distribución de subclases de IgG similar a la del plasma y libres de contaminantes, difiriendo en las cantidades de IgA presentes en los preparados. Sólo los preparados enriquecidos en IgM poseen un mayor contenido de anticuerpos específicos contra cierto número de patógenos.

\section{Evaluación de la eficacia clínica en sepsis y shock séptico}

Varios ensayos randomizados y dos metaanálisis han sido desarrollados para evaluar la posible utilidad de preparados con inmunoglobulinas poliespecíficas (IGIV o IGMAIV), anticuerpos monoclonales y antagonistas de citoquinas en la morbilidad o mortalidad (global o específica) de pacientes con sepsis o shock séptico $^{19,20}$.

Impacto sobre la mortalidad global. La mayor evaluación sobre inmunoglobulinas ha sido reportada por Alejandria et al, en un meta-análisis que agrupa 27 ensayos randomizados adecuados, 11 de ellos con IGIV o IGMAIV, 8 con anticuerpos antiendotoxinas, y 8 con anticuerpos anti-citoquinas ${ }^{19}$. La mayor parte de los trabajos incluidos fueron realizados en pacientes adultos, 4 en neonatos y sólo uno en niños. En el subgrupo de 11 ensayos con IVIG policlonal ( 6 con adultos y 5 con niños), este meta-análisis, logró demostrar una reducción en el riesgo de muerte con un riesgo relativo (RR) de 0,64 ( $\mathrm{IC}_{95}$ 0,51-0,80), tendencia que se mantuvo al considerar sólo los trabajos de mejor calidad en su diseño (RR 0,56 $\left.\mathrm{IC}_{95} 0,40-0,79\right)^{19}$ (Figura 1).

Para los preparados poliespecíficos, el impacto en la mortalidad fue mayor con los preparados enriquecidos en $\operatorname{IgM}$ en comparación a los que contienen $\mathrm{IgG}$ con un RR de 0,48 ( $\mathrm{IC}_{95} 0,30$ 0,76) vs $0,73\left(\mathrm{IC}_{95} 0,57-0,93\right)$ (Figura 1).

Al considerar todos los tipos de inmunoglobulinas (poliespecíficas, antiendotoxinas o anticitoquinas) se observó un impacto significativo sobre la mortalidad en pacientes adultos ( RR 0,62 con un $\left.\mathrm{IC}_{95} 0,49-0,79\right)$ pero no en neonatos 
Tabla 5. Dosis utilizadas de inmunoglobulinas poliespecíficas y esquemas de administración en pacientes con sepsis

Sepsis
IGIV (Flebogamma®)

IGMAIV (Pentaglobin®)

Síndrome de shock tóxico estreptocóccico IGIV (Flebogamma®)
0,75 a $1 \mathrm{~g} / \mathrm{kg}$ repartidos en 2 a 4 días consecutivos. Habitualmente una dosis mayor el primer día.

Administración: ev a una velocidad de 0,01 a $0,02 \mathrm{ml} / \mathrm{kg} / \mathrm{min}$ durante los primeros $30 \mathrm{~min}$ y luego a $0,04 \mathrm{ml} / \mathrm{kg} / \mathrm{min}$.

En caso de intolerancia disminuir o interrumpir la velocidad de infusión.

Coste diario y total aproximado: 800.000 y 1.600.000* pesos, respectivamente.

$5 \mathrm{ml} / \mathrm{kg} /$ día por 3 días. Cada $\mathrm{ml}$ contiene $38 \mathrm{mg}$ de $\operatorname{IgG}, 6$ de $\mathrm{IgM}$ y de IgA (aproximadamente 4 frascos de $100 \mathrm{ml}$ ).

Administración: Debe ser administrado luego de lograr una temperatura ambiental o corporal y a una velocidad de infusión de 0,4 $\mathrm{ml} / \mathrm{kg} /$ hora (habitualmente 12 horas).

Coste diario y total: 460.000 y $1.400 .000 *$ pesos aproximadamente.

$2 \mathrm{~g} / \mathrm{kg}$ separados en 3 días, con una dosis mayor el primer día ( $1 \mathrm{~g} / \mathrm{kg}$ ). Administración similar a lo señalado para el producto.

Coste total: $3.200 .000 *$ pesos aproximadamente

*: Coste de adquisición sin impuestos o gastos administrativos hacia octubre del 2003 (Fuente: Servicio de Farmacia, Hospital Clínico Universidad de Chile).

Tabla 6. Indicaciones más aceptadas para el uso de inmunoglobulinas poliespecíficas en pacientes con sepsis, shock séptico o shock tóxico

\section{Condición clínica}

- Shock tóxico estreptocóccico

- Shock séptico

- Sepsis postoperatoria

\section{Criterios de inclusión}

Aislamiento de $S$. pyogenes desde hemocultivos o sitios estériles asociado a hipotensión arterial y falla multiorgánica (dos o más órganos o sistemas)

Shock séptico $\leq 24$ horas y endotoxinemia $\geq 12,5 \mathrm{pg} / \mathrm{mL}$. Los criterios de shock deben incluir al menos 5 de los siguientes criterios: sospecha clínica de septicemia, fiebre $>38,5^{\circ} \mathrm{C}$, trombocitopenia $<100.000 / \mathrm{mm}^{3}$, desviación a izquierda $>5 \%$, leucopenia $<1.500 / \mathrm{mm}^{3}$ pero $>500 / \mathrm{mm}^{3}$, congestión pulmonar, CID, hipotensión arterial (PAS $<100 \mathrm{mmHg}$ ), taquicardia $(>120$ / $\mathrm{min})$, oliguria $(<500 \mathrm{~mL} /$ día $)$.

Pacientes quirúrgicos con sepsis y un puntaje $\geq 20$ puntos (sepsis score) con sepsis, aunque este último grupo sólo tiene trabajos con inmunoglobulinas poliespecíficas. (RR 0,70 con un $\mathrm{IC}_{95}$ 0,42-1,18).

Posteriormente, un meta-análisis de Olhsson et $\mathrm{al}^{21}$ en pacientes neonatos con inmunoglobulinas poliespecíficas, logró demostrar en siete trabajos seleccionados que la mortalidad global tenía una reducción significativa cuando este compuesto fue utilizado en casos con infección documentada (RR 0,55 $\mathrm{IC}_{95}$ 0,31-0,98) pero sólo una reducción de significación limítrofe cuando la inmunoglobulina fue utilizada en un escenario de sospe- cha de sepsis sin confirmación posterior ${ }^{21}$. Las diferencias observadas entre ambos meta-análisis pueden residir en el número de trabajos con niños incluidos (5 en el de Alejandria et $\mathrm{al}^{19}$ y 7 con evaluación de mortalidad en el de Olhsson et $\mathrm{al}^{21}$ ) con sólo 4 trabajos comunes. Por ejemplo, el grupo de Alejandria et al, desestimó dos de aquellos incluidos por el otro grupo debido a su deficiente calidad metodológica. Otra posible causa es la inclusión de ensayos con diferentes inmunoglobulinas (poliespecíficas, antiendotoxinas o anticitoquinas) en el meta-análisis de 


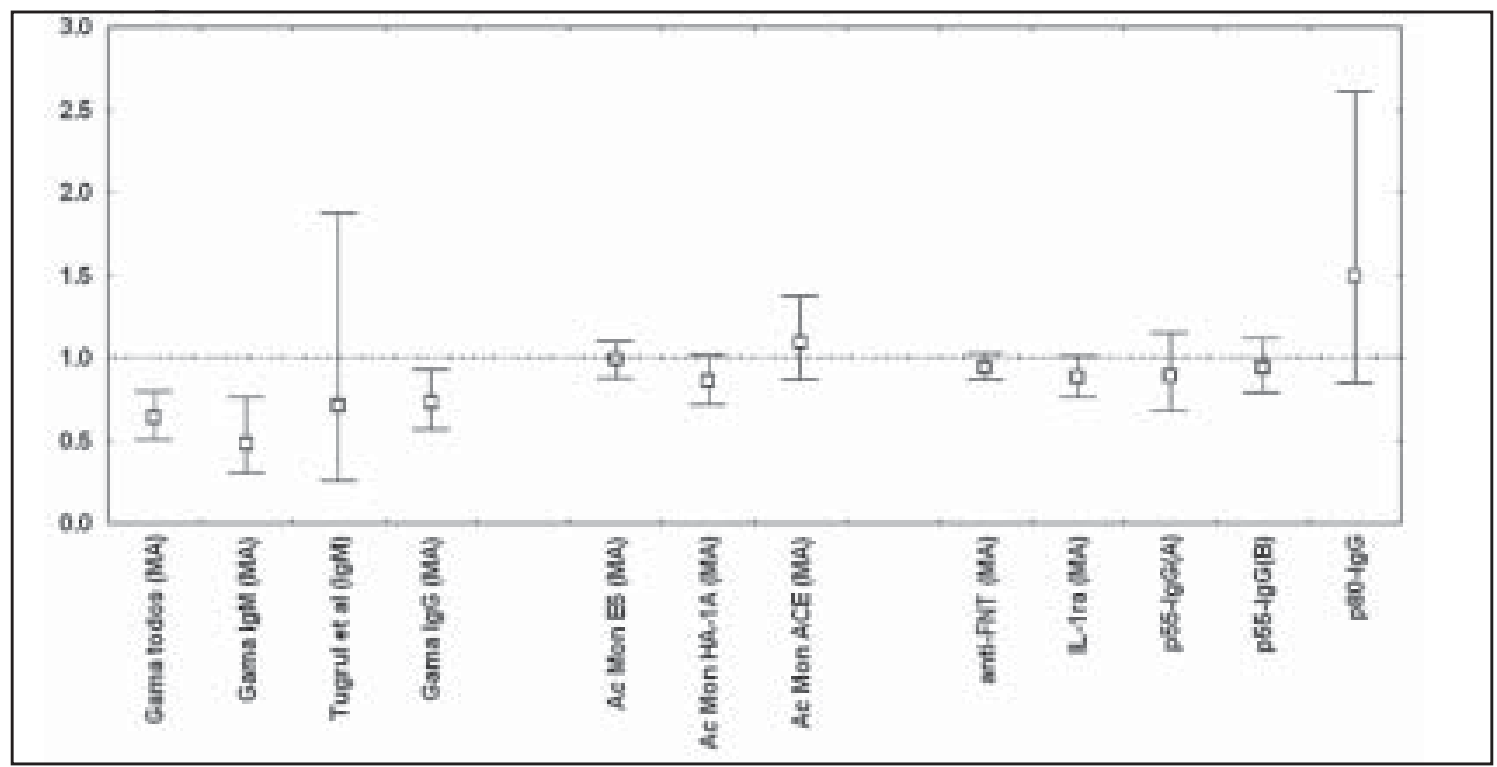

Figura 1. Riesgo relativo (RR) de la mortalidad a 28 días en sepsis o shock séptico con diferentes intervenciones inmunológicas basadas en anticuerpos o moléculas recombinantes antagonistas de citoquinas. Resultados de un metaanálisis y de otros estudios. De izquierda a derecha se presentan los valores de RR asociados a inmunoglobulinas poliespecíficas (IgG o IgG-M-A), luego los valores asociados a anticuerpos monoclonales contra estructuras bacterianas (E5, HA-1A y antígeno común de enterobacterias [ACE]), posteriormente los asociados a anticuerpos anti-FNT- $\alpha$ y al uso de antagonistas de citoquinas (IL-1 ra y proteínas de fusión Fc IgG con algunos de los receptores solubles del FNT- $\alpha$ ). El trabajo de Tugrut et al corresponde a la referencia 20. Las letras A y B señalan 2 de 3 trabajos consecutivos publicados para p55-IgG (refs. 9 y 10). La abreviación MA indica los RR obtenidos en el meta-análisis de Alejandria et al, (ref. 19). En línea discontinua se señala un RR igual a 1 que descarta un efecto protector significativo. No se incluyen en la figura los resultados del trabajo SBITS de Werdan et al (ref. 3), los resultados del trabajo de Pittet et al con proteínas de fusión como receptores solubles de FNT- $\alpha$ (ref. 12) ni aquellos asociados al uso de inmunoglobulinas en el síndrome de shock tóxico.

pacientes adultos pero sólo de inmunoglobulinas poliespecíficas en el meta-análisis de pacientes pediátricos.

Un estudio reciente desarrollado con IGIV poliespecífica y no incluido en el meta-análisis de Alejandria et al, no logró demostrar un efecto superior a placebo (estudio SBITS) $^{3}$ y por el número de pacientes contenidos $(n=653)$ podría afectar notoriamente los resultados del metaanálisis haciendo menos significativa la utilidad de estos preparados en adultos con sepsis ${ }^{3}$. De la misma manera, un estudio reciente con IGMAIV (no incluido en el meta-análisis) tampoco demostró un impacto favorable en un escaso número de pacientes con sepsis severa ${ }^{20}$. Estos nuevos antecedentes debilitan las conclusiones obtenidas en el meta-análisis de Alejandria et al, sobre el beneficio de IGIV poliespecífica enriquecida o no con IgM. (Figura 1).

Los resultados con anticuerpos monoclonales son menos claros tanto por los resultados directamente observados en los estudios originales, como lo apreciado en el meta-análisis de Alejandria et $\mathrm{al}^{19}$. En efecto, no se logró demostrar un impacto favorable al analizar por separado o en forma conjunta los estudios con el anticuerpo monoclonal E5 ( $\mathrm{n}=4$; RR 0,99 con un $\mathrm{IC}_{95}$ 0,88$1,10)$ y sólo uno de cuatro estudios con el anticuerpo HA-1A tuvo un impacto favorable sobre la mortalidad de estos pacientes ${ }^{19}$.

De la misma manera los estudios individuales de mejor calidad en forma separada o agrupados en el meta-análisis de Alejandria et al, no han logrado demostrar un impacto favorable de los anticuerpos monoclonales anti-citoquinas versus placebo ${ }^{19}$. Ensayos publicados posteriormente con un anticuerpo monoclonal dirigido hacia un antígeno común de enterobacterias (no LPS), tampoco demostraron la utilidad de esta intervención en pacientes con sepsis por bacilos gramnegativos ${ }^{6}$. De la misma manera, otros cuatro estudios desarrollados con la proteína de fusión p55-IgG o p80-IgG (receptores solubles para el FNT- $\alpha$ que actúan como antagonistas) y que no han sido incluidos en meta-análisis, no han logrado demostrar un efecto favorable sobre la morta- 
lidad global en pacientes con sepsis o shock séptico $^{9-12}$ (Figura 1).

Impacto en la mortalidad por sepsis. Sólo algunos estudios han evaluado directamente este aspecto. En el meta-análisis de Alejandria et al, tres estudios con IGIV señalaron un efecto global favorable con un $\mathrm{RR}$ de $0,35\left(\mathrm{IC}_{95} 0,18\right.$ 0,69). Dos de estos trabajos fueron desarrollados en pacientes adultos y han servido para sostener la utilidad de estos preparados a pesar de otros trabajos que no demuestran beneficio ${ }^{3,22,23}$. Estos ensayos demuestran la utilidad de una inmunoglobulina poliespecífica IgG en pacientes con sepsis postoperatoria, que cumplan ciertos criterios de gravedad o en pacientes con shock séptico de inicio reciente ( $\leq 24$ horas) con altos títulos de endotoxinemia (> 12,5 pg/mL) (Tabla 6). En sepsis postoperatoria severa se ha demostrado un beneficio en aquellos pacientes con un puntaje elevado ("sepsis score" > 20 sobre un máximo de 24) asociado a una mortalidad muy elevada $(>90 \%)^{22,24}$. Desafortunadamente, la medición de endotoxinemia no se encuentra fácilmente disponible y requiere estandarización. Por otra parte, el sistema de puntuación "sepsis score" es difícil de encontrar, no ha sido adoptado universalmente y no se ha correlacionado con otros sistemas de más amplio uso. Estos aspectos limitan la aplicación clínica de estos criterios. En el meta-análisis de Ohlsson et al no se evaluó la mortalidad específica por sepsis ${ }^{21}$.

Impacto sobre la hospitalización y otros parámetros. En los trabajos de mejor calidad metodológica no se ha logrado demostrar un efecto favorable sobre la duración de la hospitalización en pacientes adultos o neonatos ${ }^{19,21}$.

Inmunoglobulinas en el síndrome de shock tóxico. Este cuadro se caracteriza por hipotensión arterial, falla de al menos dos órganos o sistemas y la presencia de $S$. pyogenes en muestras de sangre o fluidos normalmente estériles. De aparición ocasional, se le ha asociado históricamente a fasceitis necrotizante. Tiene una alta letalidad y su aparición esporádica ha impedido el desarrollo de estudios controlados. Los diferentes meta-análisis comentados no han incluido en general pacientes de este tipo por lo esporádico de su aparición y por ello merecen un análisis por separado.

Series de casos, reportes anecdóticos y un estudio contra controles históricos han dado cuenta del probable beneficio terapéutico de IGIV poliespecífica en la recuperación de estos pacientes. Diferentes series clínicas han señalado una mejoría clínica con su uso en pacientes con infecciones invasoras y que han incluido casos con shock tóxico, fasceitis necrotizante o miositis ${ }^{25}$.
La evidencia más importante que apoya el uso de IGIV en esta grave condición proviene de dos trabajos publicados en los últimos años. El primero de ellos publicado en 1999, de tipo casocontrol, logró demostrar un incremento en la sobrevida desde aproximadamente 33 a $66 \%$ con IGIV, constituyendo el tratamiento con este preparado un factor independiente y significativo para la sobrevida de estos pacientes ${ }^{26}$.

El segundo trabajo, un estudio randomizado doble ciego publicado el 2003, demostró una reducción notable aunque no significativa, en la mortalidad y morbilidad de pacientes con síndrome de shock tóxico cuando son tratados con $\mathrm{IGIV}^{27}$. Este ensayo no logró, sin embargo, reclutar un número suficiente de pacientes después de más de dos años por lo esporádico de su ocurrencia ${ }^{27}$. Es probable que un trabajo con un número de muestra suficiente jamás sea posible y por ello muchos expertos sostienen que en esta condición la evidencia es ya suficiente para respaldar su uso y que no es posible plantear nuevos ensayos por razones éticas ${ }^{27}$. En Chile, se ha expandido progresivamente el uso en esta condición y tal vez constituya la indicación más acep$\operatorname{tada}^{28}$ (Tabla 6).

Los pacientes con shock tóxico estreptocóccico, han sido evaluados con el preparado no enriquecido (IGIV) ${ }^{26,27}$ y no se conoce la eficacia de IGMAIV en ellos.

Síntesis sobre los estudios de eficacia clínica. Ha sido difícil demostrar hasta ahora un impacto clínico favorable para las inmunoglobulinas en los tres parámetros analizados: mortalidad global, mortalidad por sepsis y duración de la hospitalización. La evidencia sobre el beneficio de esta terapia en la mortalidad global de pacientes adultos con sepsis aparece al agrupar el conjunto de los preparados o al analizar pacientes adultos o neonatos que reciben IGIV y especialmente IGMAIV. Este beneficio desaparece cuando se analizan anti-citoquinas y anti-endotoxinas o resultan discordantes para pacientes neonatos obtenidos por diferentes meta-análisis. En el caso de la mortalidad específica por sepsis en adultos hay una evidencia favorable pero limitada a un escaso número de pacientes hasta ahora y restringida a pacientes con sepsis postoperatoria grave. No existe respaldo para utilizar estos preparados como una forma de acortar la estadía hospitalaria. Aunque imperfecta, la evidencia para usar inmunoglobulinas poliespecíficas en pacientes con síndrome de shock tóxico, aparece coherente y beneficiosa al analizar series clínicas, estudios caso-control y el único ensayo randomizado disponible. 


\section{Aspectos farmacoeconómicos}

Hasta el momento, sólo se dispone de un bajo número de evaluaciones farmacoeconómicas sobre las inmunoglobulinas en el manejo de pacientes con sepsis o shock séptico y éstas incluyen sólo componentes monoclonales ${ }^{29,30}$. La validez de estas evaluaciones es cuestionable debido a que están basadas en un solo ensayo clínico que describe un efecto favorable para el anticuerpo monoclonal HA-1A, que contrasta con la ausencia de un efecto beneficioso descrito en otros ensayos $^{31-33}$.

En estos estudios, la estrategia de aplicar un tratamiento con el anticuerpo monoclonal HA-1A sólo en los pacientes con shock séptico aparece más eficiente que una aplicación sobre todos los pacientes con sepsis ${ }^{17}$. Ello se explica porque el trabajo utilizado como referencia para hacer el cálculo describe una eficacia superior de la inmunoglobulina sobre los pacientes en shock séptico y además no tiene efecto sobre pacientes con sepsis por microorganismos grampositivos. La aplicación indiscriminada sobre todos los pacientes no permite focalizar su eficacia, incrementando innecesariamente los $\operatorname{costos}^{29}$.

\section{Conclusiones}

La utilidad de las inmunoglobulinas policlonales o monoclonales en pacientes con sepsis o shock séptico continua siendo un tema polémico a pesar de los numerosos estudios desarrollados en la materia. Sólo ocasionalmente estos trabajos han demostrado directamente un beneficio y para ciertos parámetros, éste sólo ha emergido de meta-análisis.

La evidencia disponible hasta el momento sólo permite sostener que los preparados con inmunoglobulina poliespecífica $\mathrm{G}$ podrían tener un impacto favorable sobre la mortalidad global en estos pacientes y que ese impacto es más marcado para los preparados enriquecidos con IgM. Ellas pueden ser especialmente consideradas para disminuir la mortalidad (global o por sepsis), en poblaciones definidas de pacientes adultos y que incluyen a aquellos con sepsis postoperatoria con ciertos criterios de gravedad, o en pacientes con shock séptico de inicio reciente asociados a altos títulos de endotoxinemia.

De la misma manera, muchos expertos sostienen que a pesar de que no hay estudios randomizados con una ventaja significativa, la inmunoglobulina $\mathrm{G}$ poliespecífica es una herramienta de demostrado beneficio terapéutico en el manejo de pacientes con síndrome de shock tóxico estreptocóccico.

No se ha podido demostrar aún un beneficio convincente con anticuerpos monoclonales dirigidos contra diferentes estructuras bacterianas o mediante la administración de antagonistas de las citoquinas involucradas en estos cuadros.

Estas alternativas terapéuticas representan modalidades de alto costo y no se dispone aún de evaluaciones convincentes que indiquen que representan intervenciones costo-efectivas.

\section{Resumen}

La eficacia clínica de las inmunoglobulinas poliespecíficas o anticuerpos monoclonales para tratar pacientes con sepsis severa o shock séptico es aún motivo de controversia después de haberse desarrollado numerosos ensayos clínicos. Sólo algunos de ellos han podido demostrar un beneficio directo para reducir la mortalidad o este efecto es evidente tras un meta-análisis. La evidencia sostiene que las inmunoglobulinas $\mathrm{G}$ poliespecíficas reducen la mortalidad en estos pacientes, siendo este efecto mayor para las inmunoglobulinas enriquecidas con IgM. Las mejores indicaciones son sepsis postquirúrgicas o pacientes en shock séptico precoz con altos títulos de endotoxinemia. Se recomienda también indicar inmunoglobulinas intravenosas en el tratamiento de pacientes con shock tóxico estreptocóccico, evidencia establecida a través de estudios casocontrol y un ensayo clínico randomizado que mostró una clara tendencia al beneficio. La evidencia no apoya a un impacto favorable en mortalidad para anticuerpos monoclonales dirigidos contra lipopolisacáridos bacterianos, otros antígenos bacterianos o contra FNT- $\alpha$. Más aún, la infusión de antagonistas del receptor recombinante de IL-1 o receptores solubles de FNT- $\alpha$ que pudieran atenuar la respuesta inflamatoria, no han demostrado utilidad después de numerosos ensayos clínicos. Estas herramientas terapéuticas se caracterizan por un alto costo de adquisición y aún no se han realizado análisis costoefectividad.

\section{Bibliografía}

1.- Fisher C J, Slotman G J, Opal S M, Pribble J P, Bone R C, Emmanuel G, et al. Initial evaluation of human recombinant interleukin-1 receptor antagonist in the treatment of sepsis syndrome: A randomized, open-label, placebo-controlled multicenter trial. Crit Care Med 1994; 22: 12-21.

2.- Thampakkul S, Ballow M. Replacement intravenous immune serum globulin therapy in patients with antibody immune deficiency. Immunol Allergy Clin North Am 2001; 21: 165-84. 
3.- Werdan K. Intravenous immunoglobulin for prophylaxis and therapy of sepsis. Curr Opin Crit Care 2001; 7: 354-1.

4.- Sacher R. Intravenous immunoglobulin consensus statement. J Allergy Clin Immunol 2001; 108: S13946.

5.- Ballow M. Mechanisms of action of intravenous immune serum globulin in autoimmune and inflammatory diseases. J Allergy Clin Immunol 1997; 100: 151-7.

6.- Albertson T E, Panaceck E A, MacArthur R D, Jonson S B, Benjamín E, Matuschak G M, et al. Multicenter evaluation of a human monoclonal antibody to Enterobacteriaceae common antigen in patients with Gram-negative sepsis. Crit Care Med 2003; 31: 419-27.

7.- Reinhart K, Wiegand-Lohnert C, Grimminger F, Kaul M, Withington S, Treacher D, et al. Assesment of the safety and efficacy of the monoclonal antitumor necrosis factor antibody-fragment, MAK 195F, in patients with sepsis and septic shock: A multicenter, randomized, placebo-controlled, dose-ranging study. Crit Care Med 1996; 24: 733-42.

8.- Fisher C J, Dhainaut J F, Opal S M, Pribble J P, Balk R A, Slotman G J, et al. Recombinant human interleukin 1 receptor antagonist in the treatment of patients with sepsis syndrome. JAMA 1994; 271: 183643.

9.- Abraham E, Glauser M P, Butler T, Garbino J, Gelmont D, Larrere P F, et al. p55 tumor necrosis factor receptor fusion protein in the treatment of patients with severe sepsis and septic shock. A randomized controlled multicenter trial. JAMA 1997; 277: $1531-8$.

10.- Abraham E, Laterre P F, Garbino J. Lenercept (p55 tumor necrosis factor receptor fusion protein) in severe sepsis and early septic shock: A randomized, doubleblind, placebo-controlled, multicenter phase III trial with 1342 patients. Crit Care Med 2001; 29: 503-10.

11.- Fisher C J Jr, Agosti J M, Opal S M, Lowry S F, Balk R A, Sadoff J C, et al. Treatment of septic shock with the tumor necrosis factor receptor: Fc fusion protein. N Engl J Med 1996; 334: 1697-702.

12.- Pittet D, Harbarth S, Suter P M, Reinhart K, Leighton A, Barker C, et al. Impact of immunomodulating therapy on morbidity in patients with severe sepsis. Am J Respir Crit Care Med 1999; 160: 852-7.

13.- Abraham E, Wunderinck R, Silverman H, Perl T M, Nasraway S, Levy $\mathrm{H}$, et al. Efficacy and safety of monoclonal antibody to human tumor necrosis factor in patients with sepsis syndrome. JAMA 1995; 273: 934-41.

14.- Bone R C, Balk R A, Fein A M, Perl T M, Wenzel R P, Reines H D, et al. A second large controlled clinical study of E5, a monoclonal antibody to endotoxin: Results of a prospective, multicenter, randomized, controlled trial. Crit Care Med 1995; 23: 994-1006.

15.- Greenman R L, Schein R M, Martin M A, Wenzel R P, MacIntyre N R, Emmanuel G, et al. A controlled clinical trial of E5 murine monoclonal $\operatorname{IgM}$ antibody to endotoxin in the treatment of gram-negative sepsis. JAMA 1991; 266: 1097-102.

16.- Cohen J, Carlet J, INTERSEPT Study Group. INTERSEPT: An international, multicenter, placebocontrolled trial of monoclonal antibody to human tu- mor necrosis factor- $\alpha$ in patients with sepsis. Crit Care Med 1996; 24: 1431-40.

17.- Long R, Gardam M. Tumour necrosis factor-alpha inhibitors and the reactivation of latent tuberculosis infection. Can Med Assoc J 2003 Apr 29; 168: 1153-6.

18.- Gómez-Reino J J, Carmona L, Valverde V R, Mola E M, Montero M D; BIOBADASER Group. Treatment of rheumatoid arthritis with tumor necrosis factor inhibitors may predispose to significant increase in tuberculosis risk: a multicenter active-surveillance report. Arthritis Rheum 2003; 48: 2122-7.

19.- Alejandria M M, Lansang M A, Dans L F, Mantaring J B V. Intravenous immunoglobulin for treating sepsis and septic shock. Cochrane review. In: The Cochrane Library, Issue 3, 2003, Oxford: Update Software.

20.- Tugrul S, Ozcan P E, Akinci O, Seyhun H, Cagatay A, Cakar N, et al. The effects of IgM-enriched immunoglobulin preparations in patients with severe sepsis [ISRCTN28863830]. Crit Care 2002; 6: 357-62.

21.- Olhsson A, Lacy J B. Intravenous immunoglobulin for suspected or subsequently proven infection in neonates. Cochrane review. In: The Cochrane Library, Issue 4, 2002, Oxford: Update Software.

22.- Dominioni L, Dionigi R, Zanello M, Chiaranda M, Dionigi R, Acquarolo A, et al. Effects of high-dose IgG on survival of surgical patients with sepsis score of 20 or greater. Arch Surg 1991; 126: 236-40.

23.- Schedel I, Dreikhausen U, Nentwig B, Hockenschnieder M, Rauthmann D, Balikcioglu S, et al. Treatment of Gramnegative septic shock and an immunoglobulin preparation: A prospective randomized clinical trial. Crit Care Med 1991; 10: 1104-13.

24.- Dominioni L, Diogini R, Zanello M, Monico R, Cremaschi R, Dionigi R, et al. Sepsis score and acutephase protein response as predictors of outcome in septic surgical patients. Arch Surg 1987; 122: 141-6.

25.- Norrby-Teglund A, Ihendyane N, Darenberg J. Intravenous immunoglobulin adjunctive therapy in sepsis, with special emphasis on severe invasive group A streptococcal infections. Scand J Infect Dis 2003; 35: $683-89$.

26.- Kaul R, McGeer A, Norrby-Teglund A, Kotb M, Schwartz B, O'Rourke K, et al. Intravenous immunoglobulin therapy for streptococcal toxic shock syndrome. A comparative observational study. Clin Infect Dis 1999; 28: 800-7.

27.- Darenberg J, Ihendyane N, Sjolin J, Aufwerber E, Haidl S, Follin P, et al. Intravenous immunoglobulin G therapy in streptococcal toxic shock syndrome: a European randomized, double-blind, placebo-controlled trial. Clin Infect Dis 2003; 37: 333-40.

28.- Fica A, Ulloa M T, Llanos C, Luzoro A, Porte L. Diversidad clínica de las infecciones invasoras por Streptococcus pyogenes y asociación con el superantígeno SpeA. Revista Hospital Clínico Universidad de Chile 2000; 11: 9-15.

29.- Badia X, Segu L, Garcia Alonso F, Rovira J. A costefficacy analysis of treatment with antiendotoxin monoclonal antibodies in gram-negative sepsis. Med Clin (Barc) 1993; 100: 84-9.

30.- Schulman K A, Glick H A, Rubin H, Eisenberg J M. Cost-efectiveness of HA-1A monoclonal antibody for gram-negative sepsis. Economic assessment of a new therapeutic agent. JAMA 1991; 266: 3466-31. 
31.- Ziegler E J, Fisher C J Jr, Sprung C L, Straube R C, Sadoff J C, Foulke G E, et al. Treatment of gramnegative bacteremia and septic shock with HA-1A human monoclonal antibody against endotoxin: A randomized double-blind, placebo-controlled trial. N Engl J Med 1991; 324: 429-36.

32.- Derkx B, Wittes J, McCloskey R. Randomized, placebo-controlled trial of HA-1A, a human mono- clonal antibody to endotoxin, in children with meningococcal septic shock. European Pediatric Meningococcal Septic Shock Trial Study Group. Clin Infect Dis 1999; 28: 770-7.

33.- MacCloskey R V, Straube R C, Sanders C, Smith S M, Smith C R. CHESS Trial study group. Treatment. Treatment of septic shock with human monoclonal antibody HA-1A. Ann Intern Med 1994; 121: 1-5.

Correspondencia:

Alberto Fica Cubillos

afica@ns.hospital.uchile.cl 